\title{
Atomtronic Matter-Wave Lensing
}

\author{
Saurabh Pandey $\odot,{ }^{1,2}$ Hector Mas, ${ }^{1,3}$ Georgios Vasilakis, ${ }^{1}$ and Wolf von Klitzing ${ }^{1, *}$ \\ ${ }^{1}$ Institute of Electronic Structure and Laser, Foundation for Research and Technology-Hellas, Heraklion 70013, Greece \\ ${ }^{2}$ Department of Materials, Science and Technology, University of Crete, Heraklion 70013, Greece; \\ Physics Division, Los Alamos National Laboratory, Los Alamos, New Mexico 87545, USA \\ ${ }^{3}$ Department of Physics, University of Crete, Heraklion 70013, Greece; \\ Jet Propulsion Laboratory, California Institute of Technology, Pasadena, California 91109, USA
}

(Received 8 August 2020; revised 5 January 2021; accepted 16 March 2021; published 28 April 2021)

\begin{abstract}
In this Letter, we demonstrate magnetogravitational matter-wave lensing as a novel tool in atom-optics in atomtronic waveguides. We collimate and focus matter waves originating from Bose-Einstein condensates and ultracold thermal atoms in ring-shaped time-averaged adiabatic potentials. We demonstrate "delta-kick cooling" of Bose-Einstein condensates, reducing their expansion energies by a factor of 46 down to $800 \mathrm{pK}$. The atomtronic waveguide ring has a diameter of less than one millimeter, compared to other stateof-the-art experiments requiring zero gravity or free-flight distances of ten meters and more. This level of control with extremely reduced spatial requirements is an important step toward atomtronic quantum sensors.
\end{abstract}

DOI: 10.1103/PhysRevLett.126.170402

Recent years have witnessed the rise of quantum technologies from pure gedanken experiments to real applications. One of the most striking examples is atom interferometry, which employs the wavelike nature of atoms to perform, among others, extremely sensitive measurements of inertial forces such as rotation [1] and acceleration [2], making possible novel experiments in fundamental physics, e.g., a test of Einstein's equivalence principle [3] and gravitational waves detection [4]. On the applied side it enables inertial navigation, e.g., in Global Positioning System denied environments and the search for minerals and oil using gravitational mapping [5].

Today state-of-the-art atom interferometers use freely falling atoms, where the interaction time between the atoms is limited by the height of the experimental apparatus, leading to very large apparatuses, with heights extending to $10 \mathrm{~m}$ or even $100 \mathrm{~m}$ [6-11]. In order to achieve even longer interaction times some experiments have to take place in space [12-14]. Atomtronics aims at miniaturizing these atom-optical experiments and turning them into applied quantum technologies by using coherent matter-wave guides to support the atoms against gravity and thus achieve much increased interrogation times and consequently much enhanced sensitivities in a much smaller volume [15-19]. One of the key requirements of atomtronic

Published by the American Physical Society under the terms of the Creative Commons Attribution 4.0 International license. Further distribution of this work must maintain attribution to the author(s) and the published article's title, journal citation, and DOI. interferometry is that the matter waves have to be able to propagate along the waveguides without being disturbed. There have been numerous attempts at producing such waveguides using optical and magnetic potentials, e.g., in the study of the quantization of the superfluid flux [20,21] in ring-shaped magnetic [18,22-24] and optical [25,26] potentials. The main challenge of decoherence-free propagation of matter waves in waveguides has been resolved recently using time-averaged adiabatic potentials (TAAPs) as perfectly smooth waveguides, propagating condensates over distances of tens of centimeters at hypersonic speeds $[22,27]$.

In this Letter, we address one of the key challenges in atomtronics: the management of the dispersion of the atomic wave packets during their propagation in the waveguide. Just like in optical fibers, matter-wave pulses traveling in the waveguides rapidly spread with propagation distance. For Bose-Einstein condensates (BEC), this is caused by the self-interaction energy of the atoms and for thermal clouds, it is due to the velocity spread of the atoms. If left unmitigated, this makes highsensitivity interferometry difficult to implement in an atomtronic device.

We introduce atomtronic matter-wave optics as a means to manipulate the density and momentum spread of BECs and ultracold thermal atomic clouds in ultrasmooth TAAP waveguides. We use a series of time-dependent gravitomagnetic matter-wave lenses, which allow us to focus, expand, or collimate matter waves in a waveguide (see Fig. 1). We implement optimal control theory [28] and delta-kick cooling and collimation [9,29-32] as a means to minimize and manipulate the azimuthal momentum spread 
of BECs freely propagating in the ring-shaped waveguide. We can measure the resulting extremely small temperatures of only $800 \mathrm{pK}$ by free expansion in matter-wave guides over almost one second. We demonstrate a reduction of the thermal spread of a thermal cloud by a factor of 15 and of BECs by a factor of 46 . In free space, a similar reduction in energy $(\times 32)$ has been achieved by Kovachy et al. [30] and lower temperatures have been demonstrated in the $100 \mathrm{~m}$ drop tower [9] and on the space station [14]. However, in order to measure and manipulate atomic clouds at these very low temperatures, they needed the same long expansion times $(\sim 1 \mathrm{~s})$. Without the confinement afforded by matter-wave guides these values can only be achieved in microgravity or using an experimental apparatus large enough to allow the atoms to fall freely over many meters. Thanks to our ability to create extremely smooth waveguides and magnetogravitational lenses, we can achieve the same by letting the atoms expand in one dimension in a ring-shaped waveguide of one millimeter diameter.

We use ring-shaped waveguides based on TAAPs, which are formed using a combination of dc, audio-frequency, and radio-frequency (rf) magnetic fields plus gravity [27,33]: The vertically polarized, homogeneous rf field dresses the magnetic hyperfine states of the atoms in the dc magnetic quadrupole field. The ring-shaped potential results then from the time averaging induced by an audio-frequency homogeneous magnetic field oscillating in the vertical direction.

TAAPs have been shown to be very smooth with a residual roughness well below our detection limits [22]. As a consequence, the azimuthal dependence of ringshaped waveguide potentials can be fully described by $a_{1} \cos \left(\phi+\phi_{1}\right)$ and $a_{2} \cos \left(2 \phi+\phi_{2}\right)$ with $\phi$ being the azimuthal angle along the waveguide, $a_{1}$ and $a_{2}$ being arbitrary amplitudes, and $\phi_{1}$ and $\phi_{2}$ being angular positions. Higher order harmonics could arise from imperfections in the field-generating coils, but are exponentially suppressed [22,34]. By carefully adjusting the audio and rf magnetic fields, we manage to reduce the contribution of the first and second harmonic down to $250 \mathrm{nK}$ for static clouds. This induces a density modulation in moving clouds corresponding to a potential difference of $189 \mathrm{pK}$ along the full length of the ring waveguide, which we will subsequently neglect here. The resulting gravitomagnetic waveguide potential is

$$
\begin{aligned}
V_{r}(r, \phi, z)= & +\frac{1}{2} m \omega_{r}^{2}(r-R)^{2}+\frac{1}{2} m \omega_{z}^{2} z^{2} \\
& -\sin \delta \frac{1}{2} m g R \cos \left(\phi-\phi_{0}\right),
\end{aligned}
$$

where $m$ is the atomic mass, $\delta$ is the usually small angle by which the ring is tilted away from horizontal in the direction of $\phi, R$ is the radius of the ring, and $\omega_{r} / 2 \pi$ and $\omega_{z} / 2 \pi$ are the radial and axial trapping frequencies

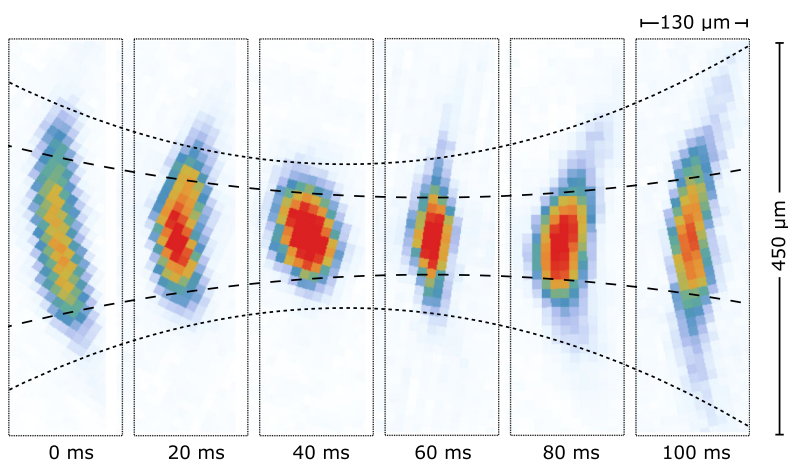

FIG. 1. Focusing of BECs and ultracold thermal clouds in a ring-shaped matter-wave guide. The absorption images were taken at different propagation times after the application of the matter-wave lens. The long-dashed line is a guide to the eye for the BECs and the dotted line for the thermal clouds. The images are taken after a time of flight of $5.3 \mathrm{~ms}$ for a ring of radius $R=485 \mu \mathrm{m}$. There were about $2 \times 10^{4}$ atoms in the BEC and $6 \times 10^{4}$ thermal atoms (BEC fraction $\sim 25 \%$ ) at a temperature of $116 \pm 33 \mathrm{nK}$. The vertical (horizontal) direction of the images is tangential (radial) to the ring at the center of the condensate. The stripes and squares are due to the pixilation of the image data.

[35]. The third term represents the gravitational potential resulting from a tilt of the waveguide by an angle $\delta$ away from horizontal [see Fig. 2(a)] [33]. The tilt angle $\delta$ and its direction $\phi_{0}$ and therefore the center of the trap can be controlled dynamically by adjusting the audio-frequency fields. The resulting time-dependent potentials can be used to accelerate a BEC in the ring and then to transport it over macroscopic distances or to manipulate BECs by applying highly controlled comoving potentials.

The principle of atomtronic matter-wave optic lensing using gravitomagnetic matter-wave lenses is outlined in Fig. 2: The atom cloud is loaded into a TAAP trap, accelerated, and released into the ring-shaped waveguide (see Supplemental Material [36]), where it expands according to its thermal or interaction energy. Some time later we apply the gravitomagnetic matter-wave lens. Depending on the strength $\delta$ and duration $\tau_{L}$ of the applied potential it can expand, focus, or collimate the atomic cloud. In atomtronic matter-wave optics, just like in photon optics, one can collimate a point source by placing it at the focus of the lens either by varying the power of the lens or the distance at which it is placed. The optical equivalent of the expansion time $\left(\tau_{0}\right)$ is the object-lens distance. The strength of the parabolic lens potential corresponds to the curvature of the refractive index of a graded-index (grin) lens, and finally the time $\tau_{L}$ corresponds to the thickness of the grin lens.

We start by studying the free expansion of thermal clouds and BECs in a ring-shaped TAAP waveguide. We load ${ }^{87} \mathrm{Rb}$ BECs from a dipole trap into the ring-shaped waveguide with the superimposed azimuthal trapping potential similar to the one that later forms the lens (see Supplemental Material [36] for BEC production, ring 
(a)
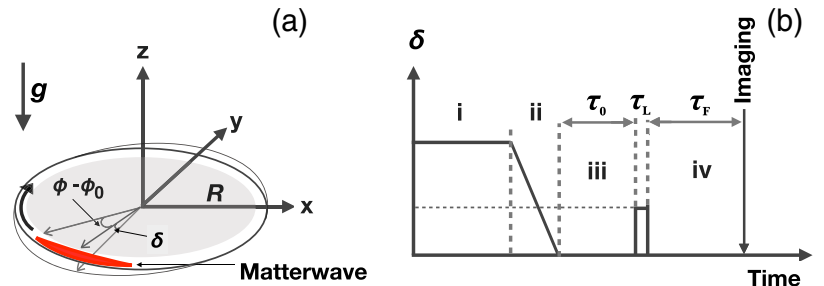

FIG. 2. (a) Schematic of the matter-wave guiding in the TAAP ring waveguide together with the azimuthal lens potential. The ring-shaped waveguide of radius $R$ is tilted away from horizontal by a small angle $\delta \ll 1$ into the direction $\phi_{0}$ with the atom clouds being centered at $\phi$. The arrow with the label $g$ indicates the direction of gravity. (b) Tilt angle $(\delta)$ of the time-averaging field during the lensing sequence: (i) BEC acceleration, (ii) adiabatic launch into the waveguide, (iii) a first free expansion of duration $\tau_{0}$ in the waveguide, followed by a delta-kick pulse of duration $\tau_{L}$, and (iv) final variable expansion in the waveguide for a duration of $\tau_{F}$. This is followed by switching off all potentials and time of flight imaging.

loading, and ring trap parameters). The measured radial, axial, and azimuthal trapping frequencies in a tilted ring trap are 85.3(4) Hz, 46.2(3) Hz, and 9.17(3) Hz, respectively [37]. We then accelerate the clouds following the bang-bang sequence of optimal control theory [22,28,36]. Within $0.2 \mathrm{~s}$, the clouds reach an angular speed of $2 \pi 10 \mathrm{rad} / \mathrm{s}(31 \mathrm{~mm} / \mathrm{s})$. We then launch the atoms into the waveguide by ramping down the tilt angle $\delta$ from $370 \mathrm{mrad}$ to $0 \mathrm{mrad}$ in $50 \mathrm{~ms}$. The clouds then rapidly expand in the azimuthal direction of the waveguide due to their temperature for the thermal clouds (or chemical potential for BECs). After some propagation time $\tau_{0}$, we switch off all confining potentials and allow the clouds to expand in 3D and image them along the $z$ direction using standard absorption imaging techniques (see Fig. 1). We determine the azimuthal expansion velocity $v=R \Delta \dot{\phi}$ by fitting the rms size of the clouds to the function

$$
\Delta l=R \sqrt{\Delta \phi_{0}^{2}+(\Delta \dot{\phi} t)^{2}}
$$

where $\Delta \phi_{0}$ is the initial rms size just when the lens is switched off. The average kinetic energy of the clouds is then $E_{\text {kin }}=1 / 2 m(R \Delta \dot{\phi})^{2}$ and $T_{\text {kin }}=2 E_{\text {kin }} / k_{B}$, where $k_{B}$ is the Boltzmann constant.

Figure 3 shows the free expansion of BECs (empty blue squares) and ultracold thermal clouds (empty red circles) in the waveguide with the dashed lines representing the fit according to Eq. (2). The azimuthal kinetic energy of the thermal clouds is found to be $T_{\mathrm{rms}}=183_{-20}^{+21} \mathrm{nK}$, which agrees nicely with the radial time-of-flight energy of $T_{\text {rad }}=181 \pm 8 \mathrm{nK}$. The azimuthal expansion energy for the BECs thus measured was $37_{-9}^{+10} \mathrm{nK}$.

We now turn our attention to atomtronic matter-wave optics using gravitomagnetic lenses. Figure 2 shows a sketch of the lensing sequence. As before, we launch the atoms into the waveguide and let them expand for some time $\left(\tau_{0}=66 \mathrm{~ms}\right)$. We then apply the gravitomagnetic lens by tilting the direction of the time-averaging field away from vertical toward the position of the atoms for a duration $\tau_{\mathrm{L}}=20.4 \mathrm{~ms}$ and tilt angle $\delta=290 \mathrm{mrad}$ (see Fig. 1). This tilts the ring and creates an azimuthal harmonic potential, which moves along the ring such that it remains centered

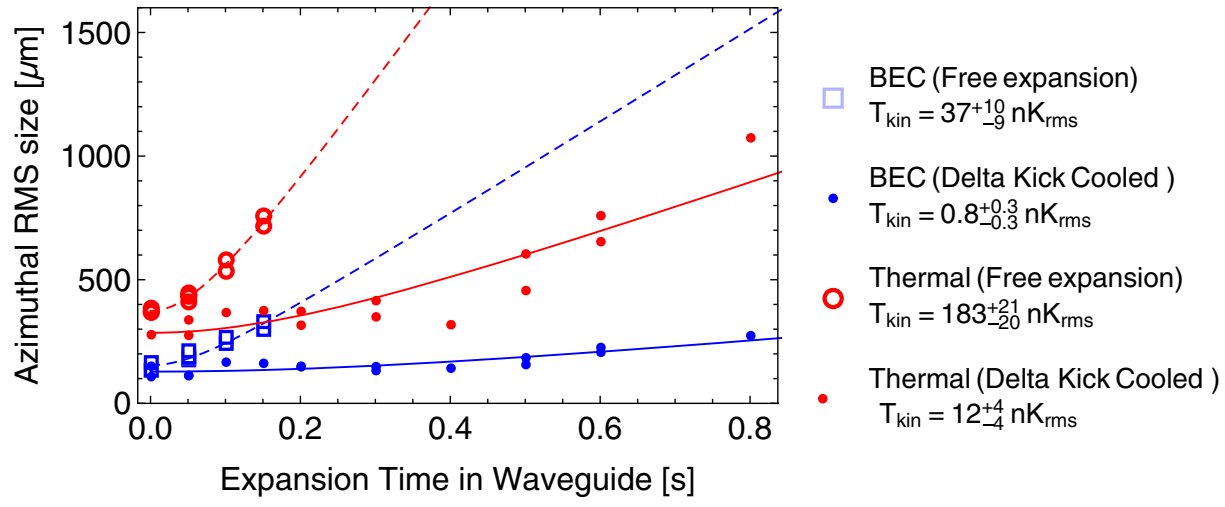

FIG. 3. Collimation of guided BECs and thermal clouds in the ring waveguide. The figure shows the azimuthal rms size of twocomponent atom clouds as they propagate freely in the ring-shaped matter-wave guide. The matter-wave lens parameters were $\tau_{L}=$ $17 \mathrm{~ms}$ and tilt $\delta=70 \mathrm{mrad}$. The thermal size is the azimuthal $1 / e$ radius $\left(R_{1 / e}\right)$ and for BECs the Thomas-Fermi radius $\left(R_{\mathrm{TF}}\right)$. The dashed lines (and empty symbols) show the free expansion of a two-component cloud in the ring-shaped waveguide in the absence of lensing. The solid lines (and full symbols) show the free expansion of a two-component cloud in the ring-shaped waveguide after nearoptimal lensing. The lines are a fit according to Eq. (2). The BECs contained about $1 \times 10^{4}$ atoms and the thermal clouds $3 \times 10^{4}$ atoms. The mean temperature measured by radial time-of-flight imaging is $187(39) \mathrm{nK}$ for the cooled thermal cloud, and $181(8) \mathrm{nK}$ for the freely expanded thermal cloud. The apparent difference in initial axial size is likely to be due to the nonzero duration of the gravitomagnetic lens (thick lens regime). The clouds are traveling at a tangential speed of $31 \mathrm{~mm} / \mathrm{s}$ in a circular waveguide of length $3.1 \mathrm{~mm}$ corresponding to more than eight round trips. 
with the atomic cloud. The strength of the lens is defined by the degree of the tilt and the duration for which it is applied. We measure the center-of-mass angular speed of the cloud after the kick pulse to be $2 \pi \times 10.07(1) \mathrm{rad} / \mathrm{s}$, while the programmed value was $2 \pi \times 10.00 \mathrm{rad} / \mathrm{s}$ (see Supplemental Material [36]). The agreement of better than $10^{-3}$ between the programmed and the measured angular speed demonstrates the extreme control afforded by matterwave optics in TAAP waveguides. No atom number loss was detected during or after the lens when compared to the no-kick case. We adjust the focus of the matter-wave lens by changing the tilt angle of the ring. The inset in Fig. 4 shows the behavior of thermal clouds for three different matter-wave lenses: For a zero tilt (green triangles) the clouds simply expand in the waveguide, whereas for a tilt of $\delta=89 \mathrm{mrad}$ (red dots) the clouds are almost collimated. Finally, for a tilt of $\delta=125 \mathrm{mrad}$ (blue squares) the cloud is focused tightly at about $100 \mathrm{~ms}$ after the lens. For different strengths of the matter-wave lens the kinetic energy of the expanding clouds changes, as depicted in the main panel of Fig. 4. By fine-tuning the strength of the gravitomagnetic lens we find the lowest kinetic energy $\left(21_{-1}^{+2} \mathrm{nK}\right)$ for a tilt of $\delta=89 \mathrm{mrad}$. The lensing has therefore reduced the initial temperature of the thermal cloud in the azimuthal direction by a factor of 6 .

In close analogy to photon waves, the focusing and collimation of matter waves is limited by spatial coherence. Therefore, thermal clouds and BECs behave quite differently with respect to matter-wave lensing, with the thermal matter waves resembling the incoherent wave fronts of incandescent light sources and BECs displaying the near

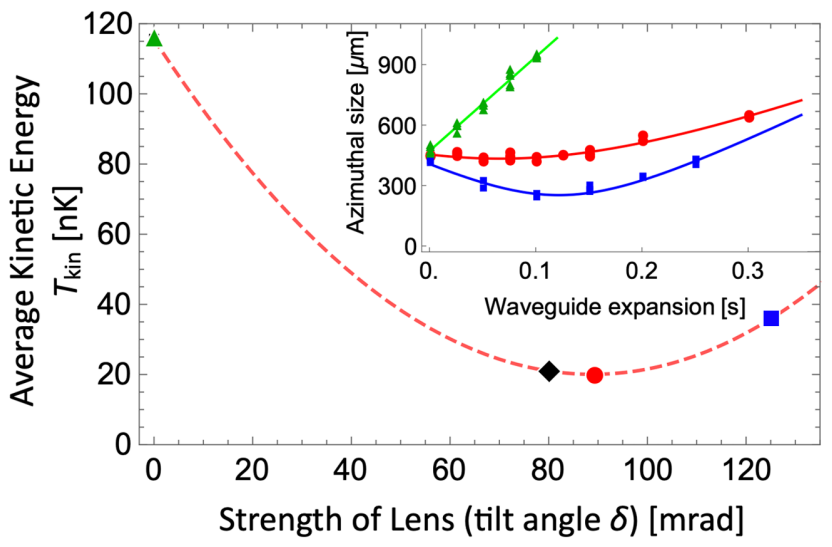

FIG. 4. The main panel shows the kinetic energy of a thermal cloud as a function of the strength of the focusing lens (the tilt angle $\delta$ of the ring-shaped waveguide keeping $\tau_{L}=17 \mathrm{~ms}$ constant). The inset shows the expansion along the waveguide for the different strengths of the lenses: no lensing ( $\delta=0$, green triangles), optimal collimation ( $\delta=89 \mathrm{mrad}$, red dots), focusing ( $\delta=125 \mathrm{mrad}$, blue squares), and near-optimal collimation ( $\delta=80 \mathrm{mrad}$, black diamond). The thermal clouds originally contain $6 \times 10^{4}$ atoms at $116_{-5}^{+5} \mathrm{nK}$. The lowest kinetic energy after atomtronic lensing in this data set is $21_{-1}^{+2} \mathrm{nK}$, corresponding to a "delta-kick cooling" by a factor of 6 . perfect wave fronts of lasers. Figure 3 shows the azimuthal expansion of thermal atom clouds and BECs traveling together in the ring-shaped waveguide. The open symbols are freely expanding whereas the solid symbols have been collimated by the gravitomagnetic lens. Allowing the clouds to freely expand and fitting Eq. (2) to the rms cloud sizes, we find average kinetic energies of $183_{-20}^{+21} \mathrm{nK}$ for the thermal cloud and $37_{-9}^{+10} \mathrm{nK}$ for the BEC. We then collimate (delta-kick cool) the atom clouds using a matterwave lens. After launching the atoms into the waveguide, we let them expand for a duration of $\tau_{0}=135 \mathrm{~ms}$. The lens has a duration of $\tau_{L}=17 \mathrm{~ms}$ and a tilt $\delta=70 \mathrm{mrad}$, which corresponds to the thin lens or the delta-kick regime where $\omega_{L} \times \tau_{L}=0.07 \ll 1$ with $\omega_{L}$ being the lens trapping frequency [30]. The solid symbols in Fig. 3 show the expansion of BECs and thermal clouds in the waveguide after the lens is turned off. A fit to Eq. (2) reveals kinetic temperature energies of $12 \mathrm{nK}$ for the thermal clouds and $800 \mathrm{pK}$ for the BECs. The kinetic energy has therefore been reduced by a factor of 15 for the thermal clouds a factor of 46 for the BECs.

The limiting factor in the final expansion energy of the BECs is the residual chemical energy at the final collimating lens, which itself is determined by the maximal size of the condensate $(\ll 2 \pi R)$ and its atom number. The final kinetic temperature could therefore be further reduced by lowering the atom number and/or the trapping frequencies. In the experiments presented here, the coldest BECs begin to approach the $1 \mathrm{D}$ regime reaching a chemical potential of $500 \mathrm{pK}$ compared to a ground state energy of 1 and $2 \mathrm{nK}$ for the radial and vertical directions, respectively [38]. It will be interesting to observe a dynamic transition between the Thomas-Fermi (TF) and 1D regimes.

In conclusion, we have demonstrated for the first time an atomtronic device with gravitomagnetic lenses. We show that the atom-optical manipulation and lensing of BECs and thermal clouds in ring-shaped waveguides can achieve a reduction in temperature by a factor of 46 , comparable to the best realized anywhere [30]. This newly found ability to generate and manipulate Bose-Einstein condensates and thermal clouds at extremely low energies together with the extreme precision of the atom-optical manipulations open the field of atomtronic devices to coherent precision manipulation and ultralow energy analysis of matter waves in simple tabletop experiments. Future applications will include dispersion control in atom-interferometry, ultralow energy collision physics, e.g., of low energy solitons, and precision experiments on Bose-Einstein condensates in one dimension. It also makes possible the generation of well-controlled vortex and Hermite-Gaussian matterwave beams.

This work has received funding from the European Union's Horizon 2020 research and innovation programme H2020-FETOPEN-2018-2019-2020-01 under Grant Agreement No. 863127 nanoLace. It was 
also supported by the project HELLAS-CH (MIS 5002735) which is implemented under the Action for Strengthening Research and Innovation Infrastructures, funded by the Operational Programme Competitiveness, Entrepreneurship and Innovation (NSRF 2014-2020) and cofinanced by Greece and the European Union (European Regional Development Fund). G. V. received funding from the European Unions Horizon 2020 research and innovation programme under the Marie Skłodowska-Curie Grant Agreement No. 750017. S. P. acknowledges financial support from the Hellenic Foundation for Research and Innovation (HFRI) and the General Secretariat and Technology (GSRT), under the HFRI Ph.D. Fellowship grant (4823).

The authors declare that they have no conflict of interest.

W. K. conceived the experiments. S. P., W. K., and H. M. designed and built the experiment, and S.P. carried them out. W. K. and S. P. analyzed the results. S. P. and W. K. wrote the manuscript. All authors edited and reviewed the manuscript.

*wvk@iesl.forth.gr

[1] T. L. Gustavson, P. Bouyer, and M. A. Kasevich, Precision Rotation Measurements with an Atom Interferometer Gyroscope, Phys. Rev. Lett. 78, 2046 (1997).

[2] A. Peters, K. Y. Chung, and S. Chu, High-precision gravity measurements using atom interferometry, Metrologia 38, 25 (2001).

[3] B. Altschul et al., Quantum tests of the Einstein Equivalence Principle with the STE-QUEST space mission, Adv. Space Res. 55, 501 (2015).

[4] S. Dimopoulos, P. W. Graham, J. M. Hogan, M. A. Kasevich, and S. Rajendran, Atomic gravitational wave interferometric sensor, Phys. Rev. D 78, 122002 (2008).

[5] M. de Angelis, A. Bertoldi, L. Cacciapuoti, A. Giorgini, G. Lamporesi, M. Prevedelli, G. Saccorotti, F. Sorrentino, and G. M. Tino, Precision gravimetry with atomic sensors, Meas. Sci. Technol. 20, 022001 (2009).

[6] M. Kasevich and S. Chu, Atomic Interferometry Using Stimulated Raman Transitions, Phys. Rev. Lett. 67, 181 (1991).

[7] D. M. Giltner, R. W. McGowan, and S. A. Lee, Atom Interferometer Based on Bragg Scattering from Standing Light Waves, Phys. Rev. Lett. 75, 2638 (1995).

[8] T. van Zoest et al., Bose-Einstein condensation in microgravity, Science 328, 1540 (2010).

[9] H. Muntinga et al., Interferometry with Bose-Einstein Condensates in Microgravity, Phys. Rev. Lett. 110, 093602 (2013).

[10] B. Canuel, A. Bertoldi, L. Amand, E. P. di Borgo, T. Chantrait, C. Danquigny, M. D. Álvarez, B. Fang, A. Freise, R. Geiger et al., Exploring gravity with the MIGA large scale atom interferometer, Sci. Rep. 8, 14064 (2018).

[11] B. Canuel et al., ELGAR - a European laboratory for gravitation and atom-interferometric research, Classical Quantum Gravity 37, 225017 (2020).
[12] D. N. Aguilera et al., STE-QUEST - test of the universality of free fall using cold atom interferometry, Classical Quantum Gravity 31, 115010 (2014).

[13] L. Badurina et al., AION: an atom interferometer observatory and network, J. Cosmol. Astropart. Phys. 05 (2020) 011.

[14] D. C. Aveline, J. R. Williams, E. R. Elliott, C. Dutenhoffer, J. R. Kellogg, J. M. Kohel, N. E. Lay, K. Oudrhiri, R. F. Shotwell, N. Yu, and R. J. Thompson, Observation of BoseEinstein condensates in an Earth-orbiting research lab, Nature (London) 582, 193 (2020).

[15] Y.-J. Wang, D. Z. Anderson, V. M. Bright, E. A. Cornell, Q. Diot, T. Kishimoto, M. Prentiss, R. A. Saravanan, S. R. Segal, and S. Wu, Atom Michelson Interferometer on a Chip Using a Bose-Einstein Condensate, Phys. Rev. Lett. 94, 090405 (2005).

[16] G. D. McDonald, C. C. N. Kuhn, S. Bennetts, J. E. Debs, K. S. Hardman, M. Johnsson, J. D. Close, and N. P. Robins, $80 \hbar k$ momentum separation with Bloch oscillations in an optically guided atom interferometer, Phys. Rev. A 88, 053620 (2013).

[17] S. Wu, E. Su, and M. Prentiss, Demonstration of an AreaEnclosing Guided-Atom Interferometer for Rotation Sensing, Phys. Rev. Lett. 99, 173201 (2007).

[18] S. Gupta, K. W. Murch, K. L. Moore, T. P. Purdy, and D. M. Stamper-Kurn, Bose-Einstein Condensation in a Circular Waveguide, Phys. Rev. Lett. 95, 143201 (2005).

[19] H. Mas, S. Pandey, G. Vasilakis, and W. von Klitzing, Bichromatic adiabatic shells for atom interferometry, New J. Phys. 21, 123039 (2019).

[20] C. Ryu, M. F. Andersen, P. Cladé, V. Natarajan, K. Helmerson, and W. D. Phillips, Observation of Persistent Flow of a Bose-Einstein Condensate in a Toroidal Trap, Phys. Rev. Lett. 99, 260401 (2007).

[21] S. Eckel, J. G. Lee, F. Jendrzejewski, N. Murray, C. W. Clark, C. J. Lobb, W. D. Phillips, M. Edwards, and G. K. Campbell, Hysteresis in a quantized superfluid 'atomtronic' circuit, Nature (London) 506, 200 (2014).

[22] S. Pandey, H. Mas, G. Drougakis, P. Thekkeppatt, V. Bolpasi, G. Vasilakis, K. Poulios, and W. von Klitzing, Hypersonic Bose-Einstein condensates in accelerator rings, Nature (London) 570, 205 (2019).

[23] A. S. Arnold, C. S. Garvie, and E. Riis, Large magnetic storage ring for Bose-Einstein condensates, Phys. Rev. A 73, 041606(R) (2006).

[24] B. E. Sherlock, M. Gildemeister, E. Owen, E. Nugent, and C. J. Foot, Time-averaged adiabatic ring potential for ultracold atoms, Phys. Rev. A 83, 043408 (2011).

[25] A. Turpin, J. Polo, Y. V. Loiko, J. Küber, F. Schmaltz, T. K. Kalkandjiev, V. Ahufinger, G. Birkl, and J. Mompart, Blue-detuned optical ring trap for Bose-Einstein condensates based on conical refraction, Opt. Express 23, 1638 (2015).

[26] T. A. Bell, J. A. P. Glidden, L. Humbert, M. W. J. Bromley, S. A. Haine, M. J. Davis, T. W. Neely, M. A. Baker, and H. Rubinsztein-Dunlop, Bose-Einstein condensation in large time-averaged optical ring potentials, New J. Phys. 18, 035003 (2016).

[27] I. Lesanovsky and W. von Klitzing, Time-Averaged Adiabatic Potentials: Versatile Matter-Wave Guides and Atom Traps, Phys. Rev. Lett. 99, 083001 (2007). 
[28] X. Chen, E. Torrontegui, D. Stefanatos, J.-S. Li, and J. G. Muga, Optimal trajectories for efficient atomic transport without final excitation, Phys. Rev. A 84, 043415 (2011).

[29] H. Ammann and N. Christensen, Delta Kick Cooling: A New Method for Cooling Atoms, Phys. Rev. Lett. 78, 2088 (1997).

[30] T. Kovachy, J. M. Hogan, A. Sugarbaker, S. M. Dickerson, C. A. Donnelly, C. Overstreet, and M. A. Kasevich, Matter Wave Lensing to Picokelvin Temperatures, Phys. Rev. Lett. 114, 143004 (2015).

[31] S. Chu, J.E. Bjorkholm, A. Ashkin, J. P. Gordon, and L. W. Hollberg, Proposal for optically cooling atoms to temperatures of the order of 10-6 K, Opt. Lett. 11, 73 (1986).

[32] M. Morinaga, I. Bouchoule, J.-C. Karam, and C. Salomon, Manipulation of Motional Quantum States of Neutral Atoms, Phys. Rev. Lett. 83, 4037 (1999).

[33] P. Navez, S. Pandey, H. Mas, K. Poulios, T. Fernholz, and W. von Klitzing, Matter-wave interferometers using TAAP rings, New J. Phys. 18, 075014 (2016).
[34] M. P. A. Jones, C. J. Vale, D. Sahagun, B. V. Hall, C. C. Eberlein, B. E. Sauer, K. Furusawa, D. Richardson, and E. A. Hinds, Cold atoms probe the magnetic field near a wire, J. Phys. B 37, L15 (2004).

[35] Typical values are $R=436 \mu \mathrm{m}, \omega_{r} / 2 \pi=85 \mathrm{~Hz}$ and $\omega_{z} / 2 \pi=46 \mathrm{~Hz}$.

[36] See Supplemental Material at http://link.aps.org/ supplemental/10.1103/PhysRevLett.126.170402 which contains the following sections: a description of BoseEinstein condensate (BEC) production, BEC loading into the ring trap, principle of BEC acceleration, and launch into the waveguide; effect of rotation on the waveguide, angular speed measurement, and calculation of the kinetic energy of BECs expanding in one dimension.

[37] Note that all errors quoted in this paper are $95 \%$ confidence intervals.

[38] The chemical potential is calculated from the size of the condensate, the transverse frequencies of the waveguide, and the number of atoms it contains. Our optical resolution is not sufficient to distinguish the $1 \mathrm{D}$ and $\mathrm{TF}$ regimes in radial time-of-flight imaging. 